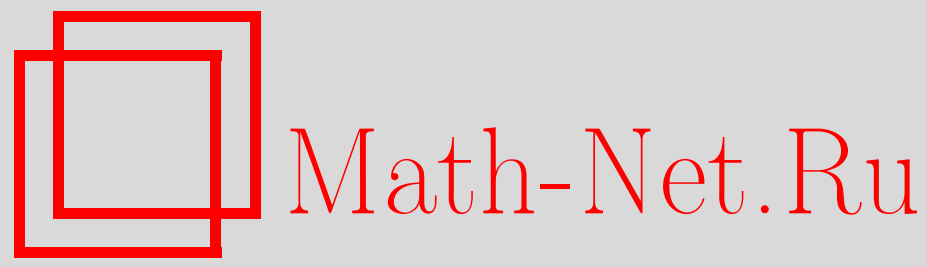

W. Bryc, A. Dembo, A. Kagan, On the maximum correlation coefficient, Теория вероятн. и ее примен., 2004, том 49, выпуск 1, 191-197

DOI: https://doi.org/10.4213/tvp246

Использование Общероссийского математического портала Math-Net.Ru подразумевает, что вы прочитали и согласны с пользовательским соглашением

http://www . mathnet.ru/rus/agreement

Параметры загрузки:

IP : 54.205 .225 .156

26 апреля 2023 г., 13:10:37

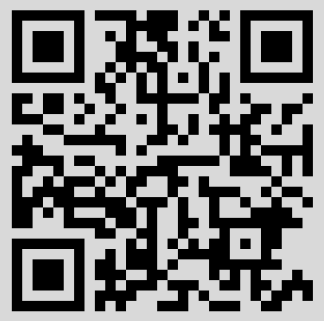


(C) 2004 г.

BRYC W.*, DEMBO A..$^{* *}$ KAGAN $A .^{* * *}$

\section{ON THE MAXIMUM CORRELATION COEFFICIENT ${ }^{1)}$}

Для произвольного случайного вектора $(X, Y)$ и независимой случайной величины $Z$ показывается, что максимальный коэффициент корреляции между $X$ и $Y+\lambda Z$ как функция $\lambda$ всюду полунепрерывен снизу и непрерывен в нуле, где и достигает своего максимума. Более того, если $Z$ принадлежит классу саморазложимых случайных величин, то максимальный коэффициент корреляции непрерывен справа и не возрастает при $\lambda \geqslant 0$ и непрерывен слева и не убывает при $\lambda \leqslant 0$. $\mathrm{He}$ зависимые случайные величины $X$ и $Z$ являются гауссовскими тогда и только тогда, когда максимальный коэффициент корреляции между $X$ и $X+\lambda Z$ равен линейной корреляции между ними. Максимальный коэффициент корреляции между суммой $n$ независимых одинаково распределенных случайных величин и суммой первых $m<n$ из них равен $\sqrt{m / n}$ (ранее этот результат был доказан только для случайных величин с конечными вторыми моментами, в этом случае максимальный коэффициент корреляции также совпадает с линейной корреляцией). Приводятся примеры, противоречашие интуитивным представлениям о поведении максимального коэффициента корреляции для $Z$ более общего вида и в пределе при $\lambda \rightarrow \infty$.

Ключевые слова и фразы: зависимость, максимальная корреляция, саморазложимые случайные величины.

1. Introduction and statement of results. The maximum correlation coefficient between two random elements $\xi, \eta$ introduced in [6] and [5] is

$$
\rho(\xi, \eta)=\sup \left\{\operatorname{corr}(\varphi(\xi), \psi(\eta)): 0<\mathbf{E}|\varphi(\xi)|^{2}<\infty, 0<\mathbf{E}|\psi(\eta)|^{2}<\infty\right\},
$$

where $\operatorname{corr}(X, Y)$ is the classical (Pearson) correlation between random variables $X$ and $Y$. The definition (1) is equivalent to

$$
\rho(\xi, \eta)=\sup \mathbf{E}\{\varphi(\xi) \psi(\eta)\}
$$

where the supremum in (2) is taken over all $\varphi, \psi$ with

$$
\mathbf{E} \varphi(\xi)=\mathbf{E} \psi(\eta)=0, \quad \mathbf{E}|\varphi(\xi)|^{2}=\mathbf{E}|\psi(\eta)|^{2}=1 .
$$

Geometrically, $\rho(\xi, \eta)$ equals the cosine of the angle between the subspaces (of a larger Hilbert space $\left.L^{2}(\xi, \eta)\right) L^{2}(\xi)=\left\{\varphi(\xi): \mathbf{E} \varphi=0, \mathbf{E}|\varphi|^{2}<\infty\right\}$ and $L^{2}(\eta)=\{\psi(\eta): \mathbf{E} \psi=0$, $\left.\mathbf{E}|\psi|^{2}<\infty\right\}$. Another well-known interpretation of $\rho$ is as the operator norm of the conditional expectation $\varphi \mapsto \mathbf{E}(\varphi(\xi) \mid \eta)$ acting on the closed subspace of $L_{2}$ consisting of functions orthogonal to constants. Thus

$$
\rho^{2}(\xi, \eta)=\sup \left\{\mathbf{E}\left\{|\mathbf{E}(\varphi(\xi) \mid \eta)|^{2}\right\}: \mathbf{E} \varphi(\xi)=0, \mathbf{E}|\varphi(\xi)|^{2}=1\right\} .
$$

The main role of $\rho(\xi, \eta)$ is that of a convenient numerical measure of dependence between $\xi$ and $\eta$. In particular, $\rho(\xi, \eta)$ vanishes if and only if $\xi$ and $\eta$ are independent.

* Department of Mathematics, University of Cincinnati, Cincinnati, OH 45221, USA; e-mail: brycw@math.uc.edu

** Department of Statistics and Department of Mathematics, Stanford University, Stanford, CA 94305, USA; e-mail: amir@math.stanford.edu

*** Department of Mathematics, University of Maryland, College Park, MD 20742, USA; e-mail: amk@math.umd.edu

1) Research of the second author was partially supported by NSF (grant DMS- 0072331). 
Explicit formulas for $\rho(\xi, \eta)$ are available in very few cases. If $(X, Z)$ is a bivariate Gaussian vector, then

$$
\rho(X, Z)=|\operatorname{corr}(X, Z)|
$$

(for a proof see, for example, [7]).

If $X=X_{1}+\cdots+X_{m}, Z=X_{1}+\cdots+X_{n}, m \leqslant n$, where $X_{1}, \ldots, X_{n}$ are independent identically distributed, nondegenerate random variables with finite second moment, then the maximum correlation between $X$ and $Z$

$$
\rho(X, Z)=\sqrt{m n^{-1}}
$$

(see [4]) and, thus, does not depend on the distribution of $X_{i}$. In Corollary 1 below, we show that (6) applies also for any independent identically distributed, nondegenerate random variables $X_{i}$. There are a few other isolated cases when $\rho(X, Y)$ is known in an explicit form. Different properties of the maximum correlation were studied in [11], [12], [9], [3], [1].

If $X, Y, Z$ are Markov-dependent, then it is easy to see that

$$
\rho(X, Y) \geqslant \rho(X, Y+Z) .
$$

Indeed, since $L^{2}(Y+Z) \subset L^{2}(Y, Z)$, one has

$$
\rho(X,(Y, Z)) \geqslant \rho(X, Y+Z) \text {. }
$$

From Markov property $\mathbf{E}(\varphi(X) \mid Y, Z)=\mathbf{E}(\varphi(X) \mid Y)$ which by (4) implies the well-known formula

$$
\rho(X, Y)=\rho(X,(Y, Z))
$$

(cf. proof of Lemma 1 in [10, p. 207]). Thus (7) follows.

If bivariate random vectors $\left(X_{1}, Y_{1}\right)$ and $\left(X_{2}, Y_{2}\right)$ are independent, then

$$
\max \left\{\rho\left(X_{1}, Y_{1}\right), \rho\left(X_{2}, Y_{2}\right)\right\} \geqslant \rho\left(X_{1}+X_{2}, Y_{1}+Y_{2}\right) .
$$

This follows from the fact that $\rho\left(X_{1}+X_{2}, Y_{1}+Y_{2}\right) \leqslant \rho\left(\left(X_{1}, X_{2}\right),\left(Y_{1}, Y_{2}\right)\right)$ and from the Csáki-Fisher identity

$$
\rho\left(\left(X_{1}, X_{2}\right),\left(Y_{1}, Y_{2}\right)\right)=\max \left\{\rho\left(X_{1}, Y_{1}\right), \rho\left(X_{2}, Y_{2}\right)\right\},
$$

see Theorem 1 in [13]. Inequality (8) yields two implications in the same spirit as (7).

(i) If $\left(X^{\prime}, Y^{\prime}\right)$ is an independent copy of $(X, Y)$, then

$$
\rho(X, Y) \geqslant \rho\left(X+X^{\prime}, Y+Y^{\prime}\right) .
$$

(ii) If $(X, Y)$ is an arbitrary bivariate random vector and $Z_{1}, Z_{2}$ are independent of each other and of $(X, Y)$, then

$$
\rho(X, Y) \geqslant \rho\left(X+Z_{1}, Y+Z_{2}\right) .
$$

We prove in this paper the following general properties of $\rho(X, Y+\lambda Z)$.

Theorem 1. The function $\lambda \mapsto \rho(X, Y+\lambda Z)$ is lower semi-continuous in $\lambda$ for any random variables $X, Y, Z$. In particular, if $Z$ is independent of the pair $(X, Y)$, then $\rho(X, Y+\lambda Z)$ is continuous at $\lambda=0$.

A random variable $Z$ is in $\mathscr{L}$ if for any $c, 0<c<1$, there exists a random variable $U_{c}$ independent of $Z$ such that

$$
Z \text { is equidistributed with } c Z+U_{c} \text {. }
$$

Equivalently, a real-valued random variable $Z$ belongs to the class $\mathscr{L}$ if its characteristic function $f(t)=\mathbf{E} e^{i t Z}, t \in \mathbf{R}$, possesses the following property: for any $c, 0<c<1$, there exists a characteristic function $f_{c}(t)$ such that

$$
f(t)=f(c t) f_{c}(t), \quad t \in \mathbf{R} .
$$

The random variables in $\mathscr{L}$ are called self-decomposable. All random variables in $\mathscr{L}$ are infinitely divisible. Necessary and sufficient conditions (in terms of Lévy functions) are known for an infinitely divisible random variable to belong to $\mathscr{L}$ (see [8, Ch. 5]). In particular, all random variables having stable distributions are in $\mathscr{L}$.

We next detail additional properties of $\rho(X, Y+\lambda Z)$ in case $Z$ is independent of $(X, Y)$ and belongs to the class $\mathscr{L}$. 
Theorem 2. If a random variable $Z \in \mathscr{L}$ is independent of a bivariate random vector $(X, Y)$, then $\lambda \mapsto \rho(X, Y+\lambda Z)$ is a nonincreasing right continuous function on $[0, \infty)$ and a nondecreasing left continuous function on $(-\infty, 0]$.

The above results hold for random elements $X, Y, Z$ taking values in an arbitrary separable Banach space; the proofs remain the same.

The following theorem is a converse of (5) holding when $X$ and $Z$ are Gaussian.

Theorem 3. If $X$ and $Z$ are independent, nondegenerate, square-integrable realvalued random variables such that for every real $\lambda$

$$
\rho(X, X+\lambda Z)=\operatorname{corr}(X, X+\lambda Z),
$$

then $X$ and $Z$ are Gaussian.

Dembo and co-authors [4] show that equality (12) may hold true for a fixed $\lambda \neq 0$ with independent, nondegenerate square-integrable non-Gaussian $X$ and $Z$, see (6).

Our following result provides the value of $\rho(X, X+\lambda Z)$ in case both $X$ and $Z$ are symmetric $\alpha$-stable random variables.

Theorem 4. Suppose $X$ and $Z$ are independent copies of an $\alpha$-stable random variable, $\alpha \in(0,2]$. Then

$$
\rho(X, X+\lambda Z)=\frac{1}{\sqrt{1+|\lambda|^{\alpha}}}
$$

for all $\lambda \geqslant 0$. If $X$ and $Z$ are symmetric, the equality (13) holds also for $\lambda<0$.

The following lemma which is key to the proof of Theorem 4, is of independent interest.

Lemma 1. Suppose $X$ and $Y$ are nondegenerate independent random variables with characteristic functions $\varphi_{X}(t)$ and $\varphi_{Y}(t)$ such that

$$
\liminf _{t \rightarrow 0} \frac{1-\left|\varphi_{Y}(t)\right|^{2}}{1-\left|\varphi_{X}(t)\right|^{2}}=c .
$$

Then

$$
\rho(X, X+Y) \geqslant \frac{1}{\sqrt{1+c}} .
$$

Suppose $X_{j}$ are independent identically distributed, nondegenerate random variables with characteristic function $\varphi(t)$ (possibly with infinite second moment). The independent random variables $X=\sum_{j=1}^{m} X_{j}$ and $Y=\sum_{j=m+1}^{n} X_{j}$ have characteristic functions $\varphi_{X}(t)=\varphi^{m}(t)$ and $\varphi_{Y}(t)=\varphi^{n-m}(t)$. Applying Lemma 1 for the pair $(X, Y)$, where $c=(n-m) / m$ by the continuity of $|\varphi(t)|^{2}$ at $t=0$, we get that

$$
\rho(X, X+Y) \geqslant \sqrt{m n^{-1}} .
$$

Combining this lower bound with the upper bound of inequality (19) of [4], we get the following corollary.

Corollary 1. The equality (6) holds for any nondegenerate independent identically distributed $X_{1}, \ldots, X_{n}$.

2. Proofs.

P r o of of $\mathrm{Th}$ e or e m 1 . Note that if $\psi_{n} \rightarrow \psi$ in $L^{2}$ and $\varphi_{n} \rightarrow \varphi$ in $L^{2}$, with both $\varphi$ and $\psi$ nonzero (in $L^{2}$ ), then $\operatorname{corr}\left(\varphi_{n}, \psi_{n}\right) \rightarrow \operatorname{corr}(\varphi, \psi)$. Consequently, in the definition (1) it suffices to consider $\varphi$ and $\psi$ in the dense subsets $B L$ of $L^{2}(\xi)$ and $L^{2}(\eta)$, consisting of bounded Lipschitz functions, using the notation $\|\psi\|_{B L}=\|\psi\|_{\infty}+\|\psi\|_{\text {Lip }}$. Fix $\varphi, \psi$ bounded and Lipschitz having positive variances at $X$ and $Y+t Z$, respectively. Define $\Delta(r)=\min (1,|r|\|Z\|)$, so

$$
|\psi(Y+t Z)-\psi(Y+s Z)| \leqslant 2\|\psi\|_{B L} \Delta(t-s),
$$

implying that

$$
\begin{gathered}
|\operatorname{cov}(\varphi, \psi(Y+t Z))-\operatorname{cov}(\varphi, \psi(Y+s Z))| \leqslant 4\|\varphi\|_{\infty}\|\psi\|_{B L} \mathbf{E}(\Delta(t-s)), \\
|\mathbf{D}(\psi(Y+t Z))-\mathbf{D}(\psi(Y+s Z))| \leqslant 8\|\psi\|_{B L}^{2} \mathbf{E}(\Delta(t-s)) .
\end{gathered}
$$


By the bounded convergence $\mathbf{E}(\Delta(t-s)) \rightarrow 0$ as $s \rightarrow t$, implying that $\mathbf{D}\{\psi(Y+s Z)\}$ is bounded away from 0 in neighborhood of $t$, so also

$$
\operatorname{corr}\{\varphi, \psi(Y+s Z)\} \rightarrow \operatorname{corr}\{\varphi, \psi(Y+t Z)\} \quad \text { as } \quad s \rightarrow t
$$

Thus

$$
\lambda \longmapsto \sup \{\operatorname{corr}(\varphi, \psi(Y+\lambda Z)): \mathbf{D}(\varphi(X)), \mathbf{D}(\psi(Y+\lambda Z)) \text { are finite }\}
$$

is lower semi-continuous. This ends the proof of the first part of the theorem.

Combining the first part of the theorem and inequality (7) for $\lambda Z$, we have

$$
\rho(X, Y) \geqslant \liminf _{\lambda \rightarrow 0} \rho(X, Y+\lambda Z) \geqslant \rho(X, Y),
$$

proving the continuity of $\rho(X, Y+\lambda Z)$ at $\lambda=0$ and completing the proof of Theorem 1 .

$\mathrm{Pr}$ o of of $\mathrm{Th}$ e or e m 2. Let $\lambda_{2}>\lambda_{1}>0$; write $\lambda_{1}=c \lambda_{2}$, where $0<c<1$. Let $U_{c}$ be a random variable independent of $X, Y, Z$ such that $Z \cong c Z+U_{c}$. From (7),

$$
\begin{aligned}
\rho\left(X, Y+\lambda_{1} Z\right) & =\rho\left(X, Y+\lambda_{2} c Z\right) \geqslant \rho\left(X, Y+\lambda_{2} c Z+\lambda_{2} U_{c}\right) \\
& =\rho\left(X, Y+\lambda_{2}\left(c Z+U_{c}\right)\right)=\rho\left(X, Y+\lambda_{2} Z\right) .
\end{aligned}
$$

If $\lambda_{2}<\lambda_{1}<0$, then setting $\lambda_{2}^{\prime}=-\lambda_{2}, \lambda_{1}^{\prime}=-\lambda_{1}$ one has

$$
\begin{aligned}
\rho\left(X, Y+\lambda_{1} Z\right) & =\rho\left(-X,-Y-\lambda_{1} Z\right)=\rho\left(-X,-Y+\lambda_{1}^{\prime} Z\right) \\
& \geqslant \rho\left(-X,-Y+\lambda_{2}^{\prime} Z\right)=\rho\left(X, Y-\lambda_{2}^{\prime} Z\right)=\rho\left(X, Y+\lambda_{2} Z\right) .
\end{aligned}
$$

The inequality (7), applied for $\lambda Z$, extends the above monotonicity properties of $\lambda \mapsto$ $\rho(X, Y+\lambda Z)$ to $[0, \infty)$ and $(-\infty, 0]$, respectively. By Theorem 1 this function is lower semicontinuous in $\lambda$, hence it is right continuous wherever nonincreasing, and left continuous wherever nondecreasing.

$\mathrm{Pr}$ o of of $\mathrm{T} \mathrm{h}$ e o r e $\mathrm{m} 3$. We may and shall assume without loss of generality that $\mathbf{E} X=\mathbf{E} Z=0, \mathbf{E} X^{2}=\mathbf{E} Z^{2}=1$. Using (12) with $\lambda=1 / s>0$ we get $\rho(X, Z+s X)=$ $\operatorname{corr}(X, Z+s X)$ and hence

$$
\mathbf{E}(X \mid Z+s X)=\frac{s}{1+s^{2}}(Z+s X)
$$

(see [4, p. 344]). Replacing $Z$ by $-Z$ in (12), it is easy to see that (17) holds also for $s \leqslant 0$. This implies

$$
\mathbf{E}[X \exp \{i t(Z+s X)\}]=\frac{s}{1+s^{2}} \mathbf{E}[(Z+s X) \exp \{i t(Z+s X)\}] .
$$

Differentiating this relation with respect to $s$ at $s=0$ we get $i t \mathbf{E}\left\{X^{2} \exp (i t Z)\right\}=$ $\mathbf{E}\{Z \exp (i t Z)\}$. Since $X, Z$ are independent and $\mathbf{E} X^{2}=1$, this shows that the characteristic function $\varphi(t)=\mathbf{E} \exp (i t Z)$ satisfies the differential equation $\varphi^{\prime}(t)=-t \varphi(t)$, and hence $Z$ is Gaussian. With $u=1 / s$, it follows from (17) that

$$
\mathbf{E}(Z \mid X+u Z)=\mathbf{E}(Z \mid Z+s X)=(Z+s X)-s \mathbf{E}(X \mid Z+s X)=\frac{u}{1+u^{2}}(X+u Z) .
$$

Reversing the roles of $X$ and $Z$, by the same argument as before $X$ is also Gaussian.

$\mathrm{Pr}$ o of of $\mathrm{L}$ e m m a 1 . Recall that for characteristic function $|\varphi(t)|^{2} \leqslant 1$. So, fixing $t \in \mathbf{R}$ such that $\left|\varphi_{X}(t)\right| \neq 1$, and considering separately the real and imaginary parts of $f(x)=e^{i t x}$, it is easy to check that (4) implies

$$
\rho^{2}(X, X+Y) \geqslant \frac{\mathbf{E}|\mathbf{E}(f(X+Y) \mid X)|^{2}-|\mathbf{E} f(X+Y)|^{2}}{\mathbf{E}|f(X+Y)|^{2}-|\mathbf{E} f(X+Y)|^{2}} .
$$

We have $|\mathbf{E} f(X+Y)|^{2}=\left|\varphi_{X}(t) \varphi_{Y}(t)\right|^{2}$,

$$
|\mathbf{E}(f(X+Y) \mid X)|^{2}=\left|\varphi_{Y}(t)\right|^{2}, \quad|f(X+Y)|^{2}=1 .
$$

Thus, if in addition $\left|\varphi_{Y}(t)\right| \neq 0$,

$$
\rho^{2}(X, X+Y) \geqslant \frac{\left|\varphi_{Y}(t)\right|^{2}\left(1-\left|\varphi_{X}(t)\right|^{2}\right)}{1-\left|\varphi_{X}(t)\right|^{2}\left|\varphi_{Y}(t)\right|^{2}}=\frac{1}{1+\left(\left|\varphi_{Y}(t)\right|^{-2}-1\right) /\left(1-\left|\varphi_{X}(t)\right|^{2}\right)} .
$$


Taking now the limsup of the right-hand side as $t \rightarrow 0$, we get the conclusion (15) out of our assumption (14).

Pro of of $\mathrm{Th}$ e or e m 4. Applying Lemma 1 to $Y=\lambda Z$ we get that

$$
\rho(X, X+\lambda Z) \geqslant \frac{1}{\sqrt{1+|\lambda|^{\alpha}}} .
$$

If $X$ and $Z$ are symmetric, the pairs $(X, X+\lambda Z)$ and $(X, X-\lambda Z)$ have the same distribution. Hence, it suffices to prove the converse of (18) for $\lambda>0$. To this end, fix $0<\varepsilon<\lambda$ and let $m<n$ be positive integers such that $\lambda-\varepsilon<(n / m-1)^{1 / \alpha}<\lambda$. Then, by Theorem 2 (and by the invariance of $\rho$ under nondegenerate linear transformations),

$$
\begin{aligned}
\rho(X, X+\lambda Z) & \leqslant \rho\left(X, X+\left(n m^{-1}-1\right)^{1 / \alpha} Z\right) \\
& =\rho\left(m^{1 / \alpha} X, m^{1 / \alpha} X+(n-m)^{1 / \alpha} Z\right)=\rho\left(S_{m}, S_{n}\right),
\end{aligned}
$$

where $S_{n}$ denotes the sum of $n$ independent copies of the $\alpha$-stable random variable $X$. Therefore, inequality (19) in [4] gives

$$
\rho(X, X+\lambda Z) \leqslant \sqrt{\frac{m}{n}} \leqslant \frac{1}{\sqrt{1+(\lambda-\varepsilon)^{\alpha}}} .
$$

Since $\varepsilon>0$ is arbitrary, this ends the proof.

3. Some counterintuitive examples. Here some examples are constructed that demonstrate counterintuitive features of the maximum correlation.

$\mathrm{E} \times \mathrm{a} \mathrm{m} \mathrm{p} \mathrm{le} 1$. Let $Z$ be a nondegenerate random variable independent of $(X, Y)$. As $|\lambda| \rightarrow \infty$, one may ask when $X$ and $Y+\lambda Z$ become «asymptotically independent», i.e.,

$$
\lim _{|\lambda| \rightarrow \infty} \rho(X, Y+\lambda Z)=0 .
$$

From [2] it follows that for bounded $\varphi, \psi$

$$
\lim _{|\lambda| \rightarrow \infty} \operatorname{cov}\{\varphi(X), \psi(Y+\lambda Z)\}=0
$$

if $Z$ has a density, and that

$$
\lim _{|\lambda| \rightarrow \infty} \operatorname{cov}\{\varphi(X), \psi(Y+\lambda Z)\}=\operatorname{cov}\{\varphi(X), \psi(Y)\}
$$

if $Z$ is discrete. The latter shows that (19) does not hold in general. Here is a related explicit example. Let $X$ be an arbitrary (nondegenerate) random variable, let the distribution of $Y$ be concentrated on $\left[-\frac{1}{2}, \frac{1}{2}\right]$ and let $Z$ be a binary random variable taking values -1 and +1 . For any (known) $\lambda$ with $|\lambda|>1, Y$ can be reconstructed from $Y+\lambda Z$ implying that for $|\lambda|>1, \rho(X, Y+\lambda Z)=\rho(X, Y)$ and does not go to 0 as $|\lambda| \rightarrow \infty$.

$\mathrm{E} \times \mathrm{m} \mathrm{ple} \mathrm{2.} \mathrm{These} X, Y, Z$ would give an example of $\rho(X, Y+\lambda Z)$ that does not decrease monotonically in $\lambda \in(0, \infty)$ unless $\rho(X, Y+\lambda Z) \equiv \rho(X, Y)$.

We shall now show that if $X=Y$ with $\mathbf{P}\left\{X=-\frac{1}{2}\right\}=\mathbf{P}\left\{X=\frac{1}{2}\right\}=\frac{1}{2}$ and $Z$ is independent of $X$ with $\mathbf{P}\{Z=-1\}=\mathbf{P}\{Z=1\}=\frac{1}{2}$, then $\rho(X, X+\lambda Z)=1 / \sqrt{2}<1$ for $\lambda=\frac{1}{2}$.

The random variable $X+Z / 2$ takes values $-1,0,+1$ with probabilities $\frac{1}{4}, \frac{1}{2}, \frac{1}{4}$, respectively. In view of (3), one may always assume

$$
\varphi\left(-\frac{1}{2}\right)=-1, \quad \varphi\left(\frac{1}{2}\right)=1 .
$$

Then $\mathbf{E}(\varphi(X) \mid X+Z / 2= \pm 1)= \pm 1$ and $\mathbf{E}(\varphi(X) \mid X+Z / 2=0)=0$. Thus $\rho^{2}=$ $\sup _{\varphi} \mathbf{E}\left\{[\mathbf{E}(\varphi(X) \mid X+Z / 2)]^{2}\right\}=\frac{1}{2}$ by (4).

$\mathrm{E} x \mathrm{a} \mathrm{m} \mathrm{p} \mathrm{l} \mathrm{e} \mathrm{3.} \mathrm{The} \mathrm{random} \mathrm{variables} X, Z$ from Example 2 are also an example of $\rho(X, X+\lambda Z)$ that, as a function of $\lambda$, is discontinuous at $\lambda=\frac{1}{2}$ and $-\frac{1}{2}$. Indeed, for any $\lambda$ with $|\lambda| \neq \frac{1}{2}, X$ can be reconstructed from $X+\lambda Z$ whence $\rho(X, X+\lambda Z)=1,|\lambda| \neq \frac{1}{2}$, while $\rho(X, X \pm Z / 2)<1$ as shown above. 
This construction can easily be generalized to $X$ and $Z$ with finite $>2$ number of values such that the continuity of $\rho(X, X+\lambda Z)$ fails to hold at prescribed $\lambda_{i}>0, i=1, \ldots, k$.

Taking $X, Z$ as above and $Y$ concentrated on $\left[-\frac{1}{6}, \frac{1}{6}\right]$, independent of $Z$, such that $1>\rho(X, Y)>1 / \sqrt{2}$, we now see by Example 1 that for $\lambda=\frac{1}{2}$,

$$
\rho(X, Y+\lambda Z)=\rho(X, Y)>\rho(X, X+\lambda Z),
$$

that is, $X$ and $Y+\lambda Z$ depend stronger than $X$ and $X+\lambda Z$ at $\lambda=\frac{1}{2}$, whereas the opposite relationship holds at $\lambda=0$.

Ex a m p l e 4. The asymptotic independence (19) may fail even when $X=Y$ and $Z$ are both in $\mathscr{L}$. Indeed, let $X$ be $\alpha$-stable random variable and let $Z$ be $\beta$-stable random variable independent of $X$, for some $0<\alpha<\beta \leqslant 2(\beta=2$ in case $Z$ is normal). With $\left(X^{\prime}, Z^{\prime}\right)$ denoting an independent copy of $(X, Z)$, the distribution of $\left(X+X^{\prime}, Z+Z^{\prime}\right)$ equals up to a nonrandom constant, that of $\left(2^{1 / \alpha} X, 2^{1 / \beta} Z\right)$. Hence, by (9),

$$
\rho(X, X+\lambda Z) \geqslant \rho\left(X+X^{\prime}, X+X^{\prime}+\lambda\left(Z+Z^{\prime}\right)\right)=\rho\left(X, X+\lambda 2^{1 / \beta-1 / \alpha} Z\right) .
$$

Since $2^{1 / \beta-1 / \alpha}<1$, Theorem 2 provides the reverse inequality, implying that $\rho(X, X+\lambda Z)$ is constant on $(0, \infty)$ and constant on $(-\infty, 0)$. By Theorem 1 this function of $\lambda$ is continuous at $\lambda=0$, hence $\rho(X, X+\lambda Z)=\rho(X, X)=1$. Obviously, (19) fails to hold in this case.

4. Open problems. I. As shown in Theorem $2, Z \in \mathscr{L}$ implies monotonicity of $\rho(X, Y+\lambda Z)$ in $\lambda$ for any $(X, Y)$ independent of $Z$.

It is interesting to find out whether the condition $Z \in \mathscr{L}$ is sufficient for continuity of $\rho(X, Y+\lambda Z)$ at $\lambda \neq 0$. More generally, what is the class of $Z$ for which monotonicity or continuity of $\lambda \mapsto \rho(X, Y+\lambda Z)$ apply for $\operatorname{all}(X, Y)$ ?

II. More generally, one may consider the properties of

$$
\rho_{p, q}(\xi, \eta)=\sup \left\{\frac{\operatorname{cov}(U, V)}{\|U\|_{p}\|V\|_{q}}: U \in L^{p}(\xi), V \in L^{q}(\eta), U, V \neq 0\right\}
$$

for $1 / p+1 / q \leqslant 1$, where $\rho=\rho_{2,2}$. Of particular interest are $\rho_{\infty, \infty}$ corresponding to strong mixing, and $\rho_{\infty, 1}$ and $\rho_{1, \infty}$ corresponding to uniform strong mixing.

\section{REFERENCES}

1. Breiman L., Friedman J.H. Estimating optimal transformations for multiple regression and correlation. [With discussion and with a reply by the authors.] - J. Amer. Statist. Assoc., 1985, v. 80, № 391, p. 580-619.

2. Bryc $W$., Smoleński $W$. On the stability problem for conditional expectation. Statist. Probab. Lett., 1992, v. 15, № 1, p. 41-66.

3. Csáki P., Fisher J. On the general notion of maximal correlation. - Magyar Tud. Akad. Mat. Kutató Int. Közl., 1963, v. 8, p. 27-51.

4. Dembo A., Kagan A., Shepp L. A. Remarks on the maximum correlation coefficient. Bernoulli, 2001, v. 7, № 2, p. 343-350.

5. Gebelein H. Das statistische Problem der Korrelation als Variations- und Eigenwertproblem und sein Zusammenhang mit der Ausgleichsrechnung. - Z. Angew. Math. Mech., 1941, v. 21, p. 364-379.

6. Hirschfeld H.O. A connection between correlation and contingency. - Proc. Cambridge Philos. Soc., 1935 , v. 31, p. 520-524.

7. Lancaster $H$. O. Some properties of the bivariate normal distribution considered in the form of a contingency table. - Biometrika, 1957, v. 44, p. 289-292.

8. Лукач E. Характеристические функции. М.: Наука, 1979, 424 с.

9. Rényi A. On measures of dependence. - Acta Math. Acad. Sci. Hungar., 1959, v. 10, p. 441-451.

10. Rosenblatt M. Markov Processes. Structure and Asymptotic Behavior. New YorkHeidelberg: Springer-Verlag, 1971, 268 p. 
11. Сарманов О.В. Максимальный коэффициент корреляции (симметричный случай). - Докл. АН СССР, 1958, т. 120, № 4, с. 715-718.

12. Сарманов О.В. Максимальный коэффициент корреляции (несимметричный случай). - Докл. АН СССР, 1958, т. 121, № 1, с. 52-55.

13. Witsenhausen H.S. On sequences of pairs of dependent random variables. - SIAM J. Appl. Math., 1975, v. 28, p. 100-113.

Поступила в редакцию

1.IV.2003

(C) $2004 \mathrm{r}$.

EICHELSBACHER P.*

\section{REFINED LARGE DEVIATIONS FOR VON MISES STATISTICS}

Приводятся достаточные условия выполнения принципа больших уклонений для вещественнозначной статистики Мизеса, улучшающие предыдущие результаты. В качестве следствия получаются достаточные условия выполнения принципа больших уклонений для $U$-статистики со значениями в банаховом пространстве. Эти условия также улучшают полученные ранее результаты.

Доказательства основаны на результатах Арконеса о больших уклонениях для случайных процессов и спектральном разложении функции ядра для статистики Мизеса и $U$-статистики соответственно.

Ключевые слова и фразы: большие уклонения, статистика Мизеса, случайные процессы.

1. Introduction. Let $(B,\|\cdot\|)$ be a real separable Banach space with a norm $\|\cdot\|$ and let $\left\{X_{i}\right\}_{i \in \mathbf{N}}$ be a sequence of independent, identically distributed random variables taking values in a measurable space $(S, \mathscr{S})$. For simplicity we assume that they are defined on the product space $(\Omega, \mathscr{A}, \mathbf{P})=\left(S^{\mathbf{N}}, \mathscr{S}^{\otimes \mathbf{N}}, \mu^{\otimes \mathbf{N}}\right)$, where $\mu$ is the distribution of $X_{1}$. Denote by $\mathscr{M}_{1}(S)$ the space of all probability measures on $(S, \mathscr{S})$. Consider for $n \geqslant m$, $n, m \in \mathbf{N}$,

$$
U_{n}(h):=U_{n}^{m}(h, \mu):=\frac{1}{\left(\begin{array}{c}
n \\
m
\end{array}\right)} \sum_{1 \leqslant i_{1}<\cdots<i_{m} \leqslant n} h\left(X_{i_{1}}, \ldots, X_{i_{m}}\right),
$$

where $h: S^{m} \rightarrow B$ is a Bochner integrable symmetric function. $U_{n}(h)$ is called a $B$-valued $U$-statistic with kernel function $h$ and degree $m$. Moreover we consider the corresponding von Mises statistic

$$
V_{n}(h):=V_{n}^{m}(h, \mu):=\frac{1}{n^{m}} \sum_{i_{1}, \ldots, i_{m}=1}^{n} h\left(X_{i_{1}}, \ldots, X_{i_{m}}\right) .
$$

In this paper we analyze the large deviations principle for these statistics.

Let us recall the definition of the large deviations principle (LDP). A sequence of probability measures $\left\{\mu_{n}\right\}_{n \in \mathrm{N}}$ on a topological space $\mathscr{X}$ equipped with $\sigma$-field $\mathscr{B}$ is said to satisfy the LDP with speed $a_{n} \downarrow 0$ and good rate function $I: \mathscr{X} \rightarrow[0, \infty]$ if the level sets $\{x: I(x) \leqslant \alpha\}$ are compact for all $\alpha<\infty$ and for all $\Gamma \in \mathscr{B}$ the lower bound

$$
\liminf _{n \rightarrow \infty} a_{n} \ln \mu_{n}(\Gamma) \geqslant-\inf _{x \in \operatorname{int}(\Gamma)} I(x)
$$

and the upper bound

$$
\limsup _{n \rightarrow \infty} a_{n} \ln \mu_{n}(\Gamma) \leqslant-\inf _{x \in \operatorname{cl}(\Gamma)} I(x)
$$

* Fakultät für Mathematik, Ruhr-Universität Bochum, NA 3/68, D-44780 Bochum, Germany; e-mail: peter.eichelsbacher@ruhr-uni-bochum.de 